\title{
Optimal Tuning of SVC-PI Controller using Whale Optimization Algorithm for Angle Stability Improvement
}

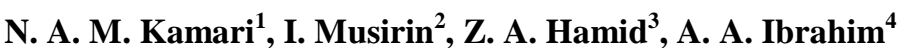 \\ ${ }^{1,4}$ Centre for Integrated Systems Engineering and Advanced Technologies (INTEGRA), \\ Faculty of Engineering and Built Environment, National University of Malaysia, Bangi, Selangor, 43600, Malaysia \\ ${ }^{2,3}$ Faculty of Electrical Engineering, Universiti Teknologi Mara, Shah Alam, Selangor, 40450, Malaysia
}

\begin{tabular}{l} 
Article Info \\
\hline Article history: \\
Received Apr 1, 2018 \\
Revised Jul 9, 2018 \\
Accepted Jul 18, 2018 \\
\hline
\end{tabular}

Keywords:

Angle Stability

PI Controller

Static Var Compensator

Whale Optimization Algorithm

\begin{abstract}
This paper proposed a new swarm-based optimization technique for tuning conventional proportional-integral (PI) controller parameters of a static var compensator (SVC) which controls a synchronous generator in a single machine infinite bus (SMIB) system. As one of the Flexible Alternating Current Transmission Systems (FACTS) devices, SVC is designed and implemented to improve the damping of a synchronous generator. In this study, two parameters of PI controller namely proportional gain, KP and integral gain, KI are tuned with a new optimization method called Whale Optimization Algorithm (WOA). This technique mimics the social behavior of humpback whales which is characterized by their bubble-net hunting strategy in order to enhance the quality of the solution. Validation with respect to damping ratio and eigenvalues determination confirmed that the proposed technique is more efficient than Evolutionary Programming (EP) and Artificial Immune System (AIS) in improving the angle stability of the system. Comparison between WOA, EP and AIS optimization techniques showed that the proposed computation approach gives better solution and faster computation time.
\end{abstract}

Copyright $\odot 2018$ Institute of Advanced Engineering and Science. All rights reserved.

\section{Corresponding Author:}

N. A. M. Kamari,

Centre for Integrated Systems Engineering and Advanced Technologies (INTEGRA),

Faculty of Engineering and Built Environment, National University of Malaysia,

Bangi, Selangor, 43600, Malaysia.

Email: azwank@ukm.edu.my

\section{INTRODUCTION}

The excitation control of generators is among the important topics in the field of power system. Flexible Alternating Current Transmission Systems (FACTS) technologies have been used widely for power systems applications [1, 2], by providing better power control and improving system flexibility and performances. Static Var Compensator (SVC) is one of FACTS devices that is connected in shunt with the system. It is a combination of a delta connected Thyristor Controlled Reactor (TCR) along with a fixed capacitor (TCRFC) in parallel with the load through a regulating transformer for controlling the bus voltage across the bus [3]. It provides a smooth, fast and precise regulation of terminal voltage. Over the last decades, many techniques have been proposed for the damping controllers for SVC to improve the damping of synchronous machines oscillations mode, by means of the lead lag controllers [4], proportional-integral (PI) controllers [5] and proportional-integral-derivative (PID) controllers [6]. In this study, PI which is one of the leading types of controllers is selected. PI calculates the error between the measured and the desired variables and tries to minimize the error by adjusting the input parameter. With the combination of SVC and PI controllers (SVC-PI), it can serve as an alternative equipment to improve angle stability and the damping performance for the power systems. For tuning the SVC-PI, two variables: proportional gain, $K_{P}$ and interval gain, $K_{I}$ are need to be optimized. 
Optimization approaches are frequently chosen to tune variables of devices in solving power system stability problems. Among them are Evolutionary Programming (EP) [7] and Artificial Immune System (AIS) [8]. EP used biological evolution process in searching for an optimal solution. On the other hand, AIS tries to benefit from the characteristics of a human immune system. A new nature-inspired meta-heuristic optimization algorithm called Whale Optimization Algorithm (WOA) is proposed [9]. This method mimics the social behavior of humpback whales which is characterized by their unique method of hunting known as the bubble-net feeding method. It brought better performance than EP and AIS in calculating the optimal solution.

This paper presents an efficient technique to determine the optimal parameters of SVC-PI damping controller in solving angle stability problems. Both $K_{P}$ and $K_{I}$ variables are determined using WOA and it was compared with EP and AIS optimization methods. The objective is to produce the most stabilized technique in the shortest time.

\section{PROBLEM FORMULATION}

In this study, the Phillips-Heffron block diagram model for the single machine infinite bus (SMIB) system that equipped with SVC and PI controller (SVC-PI) is considered. The concept of SMIB is equivalent with one synchronous machine connected to one big bus with infinite load. The input of SVC-PI is fed from the speed deviation $\Delta \omega_{r}$ and the output then channelled to $\Delta \sigma$. The block diagram model of SMIB with SVCPI is shown in Figure 1.

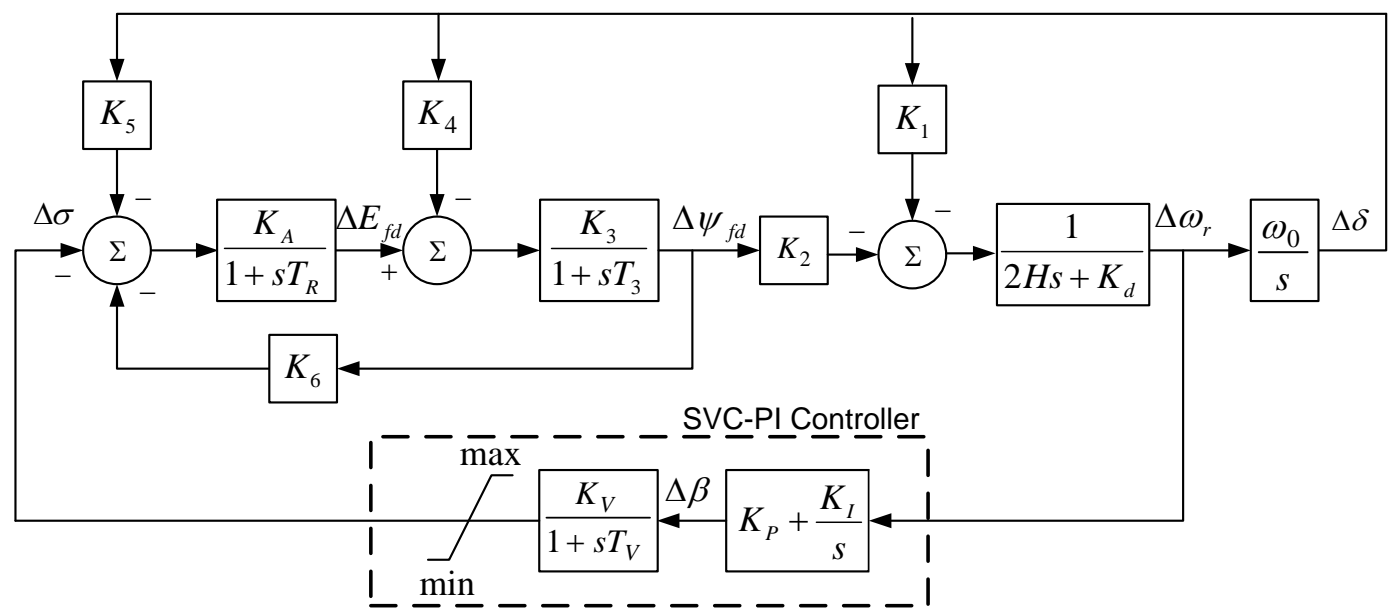

Figure 1. The block diagram model of SMIB with SVC-PI

SVC-PI can be segregated to SVC component and PI component. SVC component consists of circuit and time constant, $K_{V}$ and $T_{V}$. The PI component is derived from proportional and integral gain of the PI controller which simplified respectively as $K_{P}$ and $K_{I}$.

The equations represent SMIB system installed with SVC-PI are as followed:

$$
\begin{aligned}
& \frac{\Delta \omega_{r}}{\Delta t}=\frac{\Delta T_{m}-K_{1} \Delta \delta-K_{d} \Delta \omega_{r}-K_{2} \Delta E_{q}}{2 H} \\
& \frac{\Delta \delta}{\Delta t}=\omega_{0} \Delta \omega_{r} \\
& \frac{\Delta E_{q}}{\Delta t}=-\frac{K_{3} K_{4} \Delta \delta+\Delta E_{q}-K_{3} \Delta v_{f}}{T_{K}} \\
& \frac{\Delta v_{f}}{\Delta t}=-\frac{K_{R} K_{5} \Delta \delta+K_{R} K_{6} \Delta E_{q}+\Delta v_{f}}{T_{R}} \\
& \frac{\Delta \sigma}{\Delta t}=-\frac{\Delta \sigma-K_{V} \Delta \beta}{T_{V}}
\end{aligned}
$$




$$
\frac{\Delta \beta}{\Delta t}=\left(K_{1}+\frac{K_{d} K_{P}}{2 H}\right) \Delta \omega_{r}-\frac{K_{1} K_{P}}{2 H} \Delta \delta+\frac{K_{2} K_{P}}{2 H} \Delta E_{q}
$$

$H$ is the inertia constant, $T_{m}$ is the mechanical torque, $K_{D}$ is the damping torque coefficient, $K_{R}$ and $T_{R}$ are the circuit constant and time constant of the exciter oscillation system, respectively. $\omega_{0}$ is equal to $2 \pi f_{0}$. The $K$ constants i.e. $K_{1}, K_{2}, K_{3}, K_{4}, K_{5}, K_{6}$ and $T_{K}$ are represented the dynamic characteristics of the system model. Detail calculation of parameters $K_{1}, K_{2}, K_{3}, K_{4}, K_{5}$ and $K_{6}$ can be found in [10].

The equation $(1)-(6)$ are then combined to create a matrix form as followed:

$$
X_{S V C}^{\cdot}=A_{S V C} \cdot X_{S V C}+B_{S V C} \cdot U
$$

Where

$$
\begin{aligned}
& X_{S V C}=\left[\begin{array}{llllll}
\Delta \omega_{r} & \Delta \delta & \Delta E_{q} & \Delta v_{f} & \Delta \sigma & \Delta \beta
\end{array}\right]^{T} \\
& A_{S V C}=\left[\begin{array}{cccccc}
-K_{d} / 2 H & -K_{1} / 2 H & -K_{2} / 2 H & 0 & 0 & 0 \\
\omega_{0} & 0 & 0 & 0 & 0 & 0 \\
0 & -K_{3} K_{4} / T_{K} & -1 / T_{K} & K_{3} / T_{K} & 0 & 0 \\
0 & -K_{R} K_{5} / T_{R} & -K_{R} K_{6} / T_{R} & -1 / T_{R} & 0 & 0 \\
0 & 0 & 0 & 0 & -1 / T_{V} & -K_{V} / T_{V} \\
K_{I}-K_{P} K_{d} / 2 H & -K_{P} K_{1} / 2 H & -K_{P} K_{2} / 2 H & 0 & 0 & 0
\end{array}\right] \\
& B_{S V C}=\left[\begin{array}{llllll}
1 / 2 H & 0 & 0 & 0 & 0 & 0
\end{array}\right]^{T} \\
& U=\left[\Delta T_{m}\right]
\end{aligned}
$$

Here, $A$ and $B$ are matrices of real constants and variables with appropriate dimensions. $X$ and $U$ are the state vector and input signal vectors, respectively.

In this paper, the value of proportional gain, $K_{P}$ and integral gain $K_{I}$ of the PI controller are kept within specified limits. The WOA algorithm is proposed to calculate the optimal value of these two SVC-PI parameters. The SMIB systems parameters are shown in Table 1.

Table 1. The Parameters for SMIB and SVC System.

\begin{tabular}{cc}
\hline Components & List of Parameters \\
\hline Generator & $H=2.0, T_{d 0}=8.0, X_{d}=1.81, X_{q}=1.76, X_{d}^{\prime}=0.30$, \\
& $R_{a}=0.003, K_{s d}=K_{s q}=0.8491, E_{t}=1.0 \angle-36^{\circ}$ \\
Transmission Line & $R_{e}=0.0, X_{e}=0.65, X_{L}=0.16$ \\
Exciter and SVC & $K_{A}=100, T_{A}=0.05, K_{V}=10, T_{V}=0.05$ \\
\hline
\end{tabular}

\section{COMPUTATIONAL INTELLIGENCE METHODS}

Lately, the use of Artificial Intelligence (AI) technology is synonymous in solving power system problems. AI techniques utilize the logic and knowledge representations of expert systems, artificial neural network (ANN) [11] and evolutionary computation (EC). The EC field includes Particle Swarm Optimization (PSO) [12], Evolutionary Programming (EP) [7], Artificial Immune Systems (AIS) [8] and Firefly Algorithm (FA) [13]. In this study, the proposed WOA is compared with EP and AIS in order to highlight their merit. The algorithms for all methods are discussed below.

\subsection{Whale Optimization Algorithm}

Whale Optimization Algorithm (WOA) is a novel nature-inspired meta-heuristic optimization algorithm proposed by Seyedali Mirjalili and Andrew Lewis in 2016, which mimics the social behavior of humpback whales. The humpback whales dive deeply, create bubbles in a spiral shape around the prey, and swim to the surface during the manoeuvre. They usually attack the small fishes close to the surface. Based on this behavior, the modelling of WOA can be divided into three operators: the search for prey (exploration phase), the encircling prey, and the bubble-net foraging (exploitation phase). In this paper, the WOA works as follows:

a) Step 1 (Initialization): The whale position $x_{i}$ of $N$ solution $(i=1, \ldots, N)$ are randomly created to form initial whale population. Each whale is evaluated using the objective function $J$. In this initialization process, $J_{i}$ 
is set as personal best objective function $J_{i, p}$ for $i$ th whale. The maximum objective function of all whales

$J_{\max }$ is set as best objective function $J_{b e s t}$ and the whale position at $J_{b e s t}$ is set as best position $x_{b e s t}$.

b) Step 2 (Update positions): $x_{i}$ is updated when the following conditions are met:

1) If $p<0.5$ and $|A|<1$, update the current whale position $x_{i}$ by the following equation:

$$
x_{i}(j)=x_{\text {best }}+A \cdot\left(C \cdot x_{\text {best }}-x_{i}(j-1)\right)
$$

where $A$ is the inertia weight, $p$ and $C$ are both random functions in the range [0,1]. This phase is called encircling prey. In this phase, WOA assumes that the current best position $x_{\text {best }}$ is the target prey or close to the optimum.

2) If $p<0.5$ and $|A|>1$, search random position for the current whale position $x_{i}$ by the following equation:

$$
x_{i}(j)=x_{\text {rand }}+A \cdot\left(C \cdot x_{\text {rand }}-x_{i}(j-1)\right)
$$

This phase is called exploration phase. In this phase, the search agents are forced to move far away from the best whale position.

3) If $\mathrm{p}>0.5$, update the current whale position xi by the following equation:

$$
x_{i}(j)=x_{\text {best }}+e^{b l} \cdot \cos (2 \pi l) \cdot\left(C \cdot x_{\text {best }}-x_{i}(j-1)\right)
$$

where $b$ is a constant, $l$ is a random number in $[-1,1]$. This phase is called exploitation phase. This equation is created between the position of whale and prey to mimic the helix-shaped movement of humpback whales.

c) Step 3 (Calculate objective functions): The new $J, J_{\max }$ and the minimum objective function of all whales $J_{\min }$ are calculated.

d) Step 4 (Stopping criteria): The search process will be terminated if it reaches the maximum number of iterations or the value of $\left(J_{\max }-J_{\min }\right)$ is very close to 0 . If the process is not terminated, the iteration will be set to $j=j+1$ and algorithm will start again from Step 2 .

The overall process of WOA algorithm is illustrated in the form of flowchart, as shown in Figure 2.

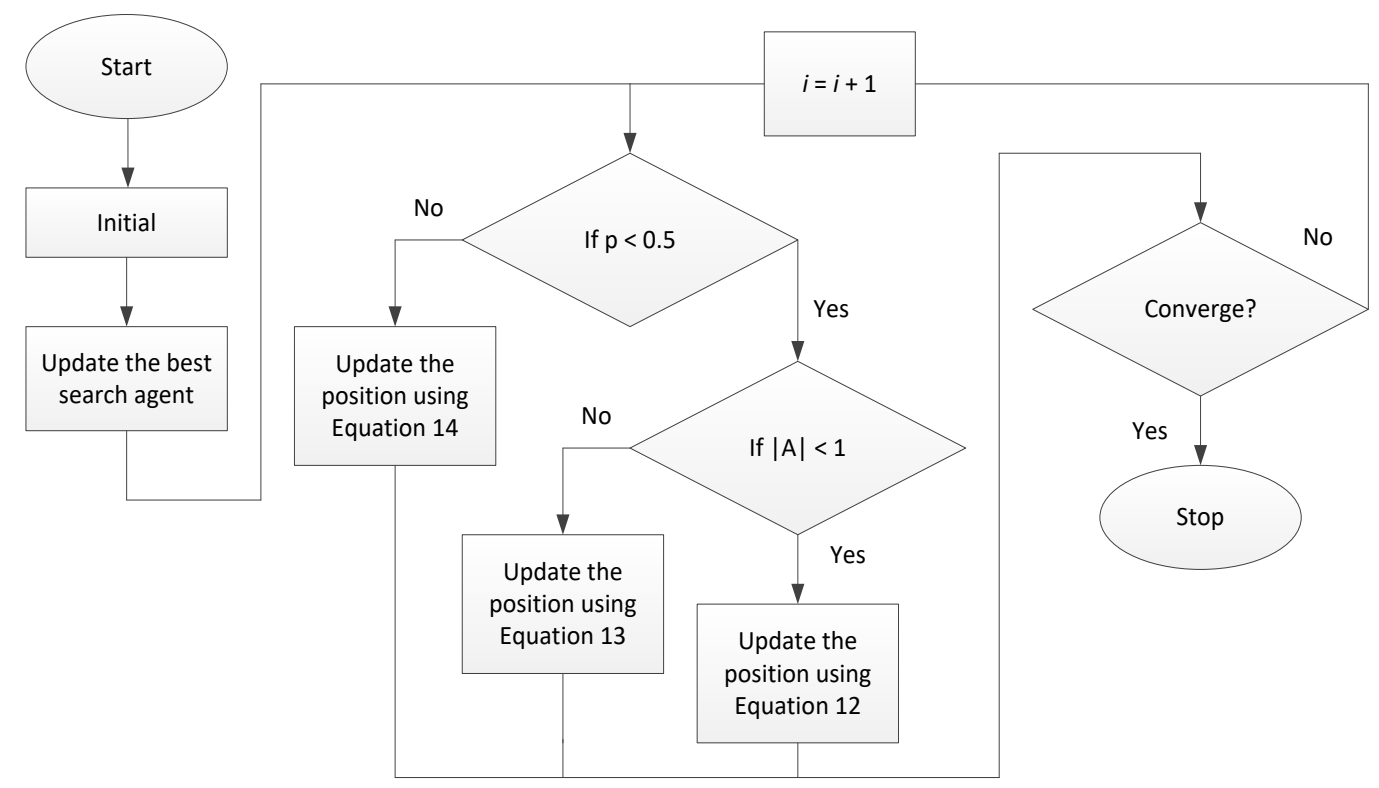

Figure 2. Flowchart for the Whale Optimization Algorithm (WOA)

\subsection{Evolutionary Programming}

The Evolutionary Programming (EP) uses the model of biological evolutionary process to determine the solution for nonlinear engineering problems. This searching process of desirable solution is based on the 
natural biological evolution. The process of EP algorithm includes initialization, statistical evaluation, fitness calculation, mutation, combination and selection. Further details about this technique can be found in [7].

\subsection{Artificial Immune System}

Artificial Immune System (AIS) and EP share many common aspects of optimization techniques. $\mathrm{EP}$ is based on the natural evolution model, while AIS tries to benefit from the characteristics of a human immune system. Basic algorithm for AIS-based optimization is called the Clonal Selection Algorithm (CSA). The algorithm of AIS that involved are initialization, statistical evaluation, fitness calculation, mutation, combination, selection and one extra component, namely cloning. The flow chart which represents AIS algorithm can be found in [8].

\subsection{Fitness Equation}

The implementation of SVC-PI in the SMIB system is capable to improve the oscillations damping and minimize the power angle deviation after a disturbance. In this paper, a fitness equation based on the minimum damping ratio $\xi_{\min }$ is proposed as follows [14]:

$$
J=\xi_{\min }=\min \left(-\frac{\sigma_{i}}{\sqrt{\sigma_{i}^{2}+\omega_{i}^{2}}}\right), \xi_{i} \in \xi_{E M}
$$

where, $\xi_{i}$ is the damping ratio of the $i^{\text {th }}$ electromechanical modes of oscillation, respectively. $\xi_{E M}$ is the set of damping ratios of the electromechanical modes of oscillation. $\sigma_{i}$ and $\omega_{i}$ are the real and imaginary part of the $i^{\text {th }}$ eigenvalue at the loading condition, respectively.

With the optimization of $\xi_{\text {min }}$, the system poles are consistently pushed further left of the imaginary $(j \omega)$ axis. Also, the decreasing value of imaginary part of the eigenvalue $\omega$ will shift eigenvalue region towards the real axis. The area of eigenvalues on the phase plan for comparison case between system with and without proposed approach is shown in Figure 3. The area bounded by this effect can be shown as a trapezoid-shaped sector on the phase plan.

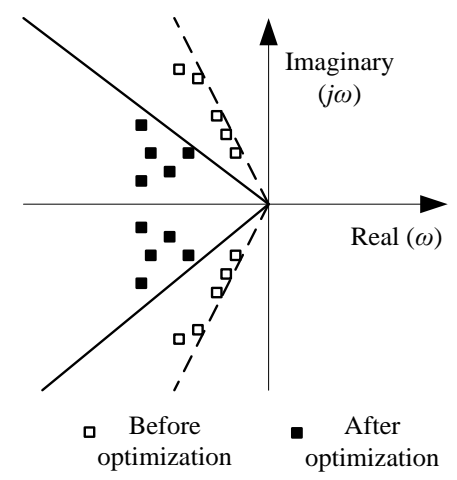

Figure 3. Comparison of eigenvalue areas on the complex s-plane (with and without $J$ ).

Therefore, the design problem can be formulated as:

Maximize $J$

This is subject to:

$$
\begin{aligned}
& K_{P}{ }^{\text {max }} \leq K_{P} \leq K_{P}{ }^{\text {min }}, \\
& K_{I}{ }^{\text {max }} \leq K_{I} \leq K_{I}{ }^{\text {min }}
\end{aligned}
$$

Here, $K_{P}$ and $K_{I}$ are optimized by EP, AIS and WOA approach. The fitness values and parameters involved in these three techniques are tabulated in Table 2. 
Table 2. The Parameters for AIS, EP and WOA Algorithms

\begin{tabular}{cccc}
\hline Methods & AIS & EP & WOA \\
\hline List of Parameters & $\beta_{a i s}=0.05$ & $\beta_{e p}=0.05$ & $A=0.9, b=1$ \\
\hline
\end{tabular}

\section{RESULTS AND DISCUSSION}

In this paper, simulation studies of SVC-PI based SMIB power system are carried out in MATLAB environment. Two parameters: proportional gain $K_{P}$ and integral gain $K_{I}$ are optimized until maximum value of the fitness equation $J$ is defined.

In this study, the performance of system with conventional SVC-PI system (C-PI) is compared to SVC-PI system optimized by WOA (WOA-PI), SVC-PI system optimized by EP (EP-PI) and SVC-PI system optimized by AIS (AIS-PI). Following two different loading conditions are simulated:

a) Case $1(\mathrm{P}=0.35$ p.u., $\mathrm{Q}=0.7$ p.u. $)$

b) Case $2(\mathrm{P}=0.15$ p.u., $\mathrm{Q}=0.3$ p.u. $)$

The response of speed deviation for Case 1 is shown in Figure 4. The system with C-PI is poorly damped and becomes stable for more than 3 seconds. For EP-PI, AIS-PI and WOA-PI, all three systems are improving the damping capability. From the speed response, its shows that WOA-PI manage to deliver the fastest and smoothest damping performance, followed by EP-PI and AIS-PI.

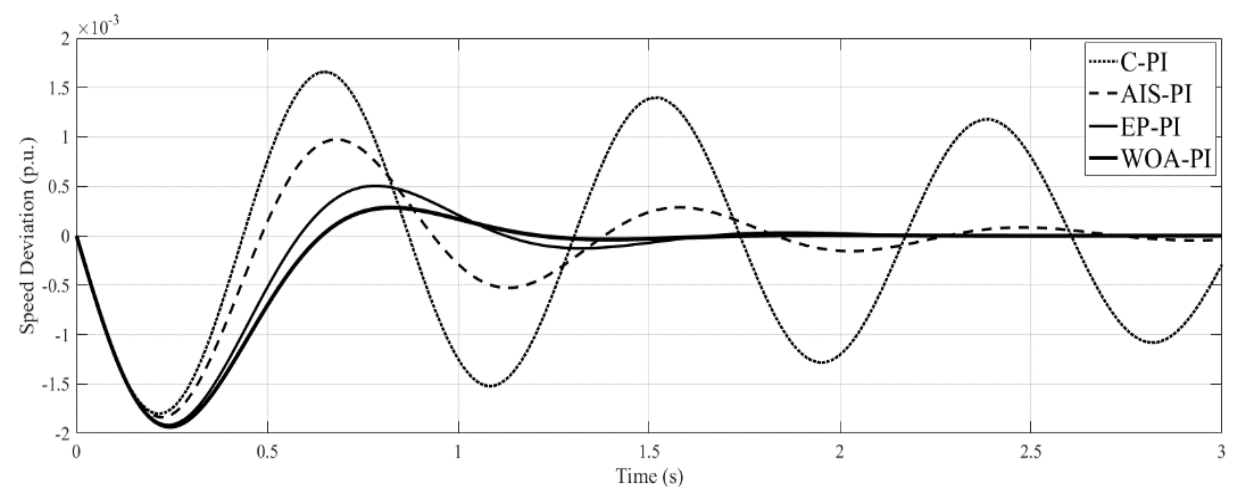

Figure 4. Speed response for Case 1

The regions of eigenvalues location in complex s-plane for all four techniques in Case 1 are shown in Figure 5. It indicates that WOA method is more capable to improve the stability of the system by pushing the eigenvalues location far further to the left-hand side of the complex s-plane and closer to the real, $\sigma$ axis. It also shows that C-PI have two eigenvalues that place near to the left-hand side of the $j \omega$ axis, indicate that the system is the most less stable.

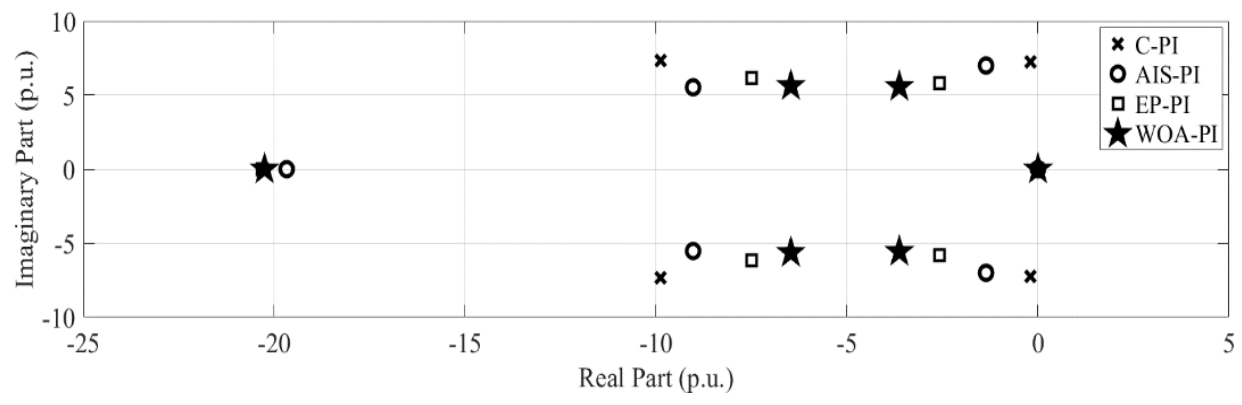

Figure 5. Complex s-plane for Case 1

The results of fitness profiles (which based on minimum damping ratio $\xi_{\min }$ ), number of iteration $N_{i}$ and computation time using C-PI, AIS-PI, EP-PI and WOA-PI for Case 1 are tabulated in Table 3. From the results, WOA-PI optimized the highest value of $J$ followed by EP-PI, AIS-PI and C-PI. From the iteration

Optimal Tuning of SVC-PI Controller using Whale Optimization Algorithm for Angle ... (N. A. M. Kamari) 
perspective, AIS is terminated in the shortest iteration, which is 4 iterations, followed by WOA with 12 iterations, while the EP was stopped at iteration 22. This shows that AIS give the shortest computation time compared to WOA and EP. Even though slower than AIS in computation time, WOA still the best approach, as it gives far more good result in improving damping capability of the system.

Table 3. Comparison of C-PI, AIS-PI, EP-PI and WOA-PI System for Case 1

\begin{tabular}{ccccc}
\hline Type & $K_{P}$ & $K_{I}$ & $J\left(\xi_{\min }\right)$ & $N_{i}$ \\
\hline C-PI & 0.5 & 3.0 & 0.0272 & - \\
AIS-PI & 0.6121 & 16.8865 & 0.1908 & 4 \\
EP-PI & 1.5090 & 25.1941 & 0.4031 & 14 \\
WOA-PI & 1.6003 & 28.0343 & 0.5462 & 12 \\
\hline
\end{tabular}

The response of speed deviation for Case 2 is shown in Figure 6. Here also, the proposed WOA-PI system shows lower oscillation and better damping compared to other four techniques. The regions of eigenvalues location in complex s-plane for Case 2 as shown in Figure 7. From the results, WOA is the most sufficient approach in shifting the eigenvalues towards $\sigma$ axis, as well as further to the left-hand side of $j \omega$ axis at the loading condition compared to other three techniques. Table 4 tabulates the results for comparative studies using C-PI, AIS-PI, EP-PI and WOA-PI for Case 2. Results obtained shows that WOA-PI achieve higher fitness compared to other three techniques.

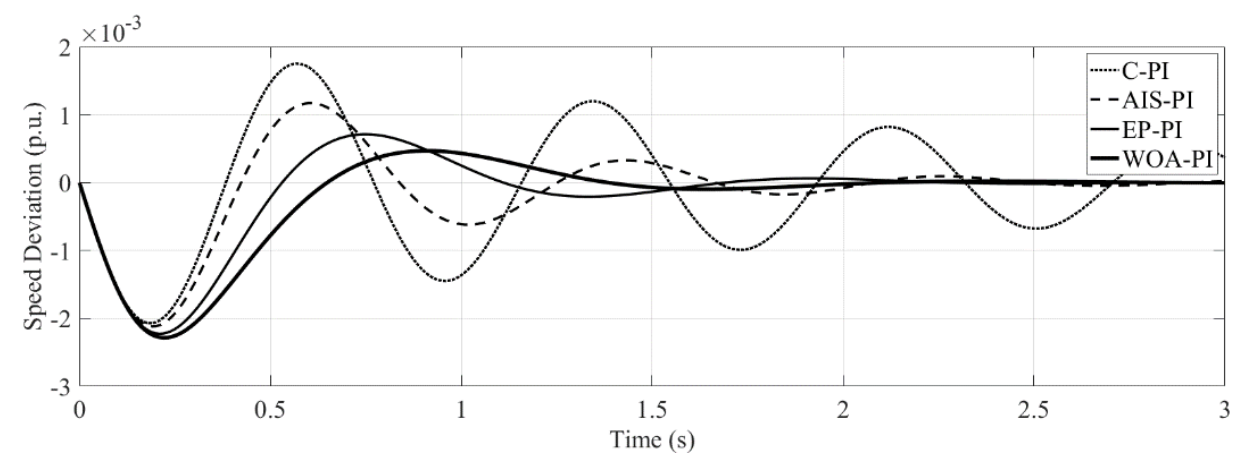

Figure 6. Speed response for Case 2

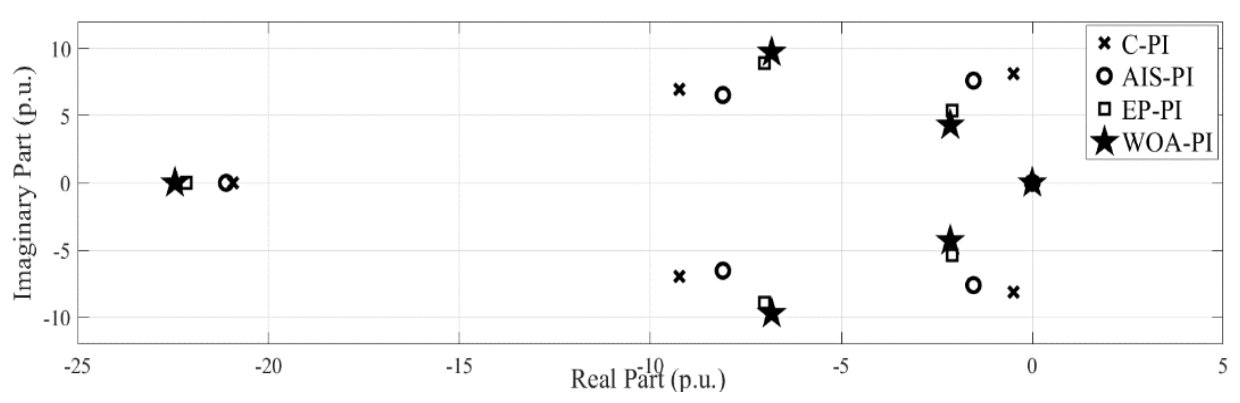

Figure 7. Complex s-plane for Case 2

Table 4. Comparison of C-PI, AIS-PI, EP-PI and WOA-PI System for Case 2

\begin{tabular}{ccccc}
\hline Type & $K_{P}$ & $K_{I}$ & $J\left(\xi_{\min }\right)$ & $N_{i}$ \\
\hline C-PI & 0.5 & 3.0 & 0.0604 & - \\
AIS-PI & 0.8119 & 7.8797 & 0.1987 & 5 \\
EP-PI & 1.5673 & 12.1472 & 0.3629 & 15 \\
WOA-PI & 1.8573 & 14.472 & 0.4472 & 10 \\
\hline
\end{tabular}

\section{CONCLUSION}

This paper proposed a new optimization approach for tuning SVC with PI controller. Three methods based on AIS, EP and WOA computation intelligence methods for optimizing $K_{P}$ and $K_{I}$ of PI controller have 
been developed. Results obtained from the study show that WOA outperformed EP and AIS in terms of giving better damping and lower oscillation. The performances are validated with respect to speed deviation response as well as minimum damping ratio $\xi_{\min }$ and eigenvalues.

\section{REFERENCES}

[1] M. M. ElAdany, A. A. ElDesouky, A. A. Sallam. Power System Transient Stability: An Algorithm for Assessment and Enhancement Based on Catastrophe Theory and FACTS Devices. IEEE Access. 2018; Early Access: 1-12.

[2] S. Chirantan, S. C. Swain, P. C. Panda, R. Jena. Enhancement of Power Profiles by Various FACTS Devices in Power System. International Conference on Communication and Electronics System. Coimbatore. 2017; 896-901.

[3] S. Das, D. Chatterjee, S. K. Goswami. Tuned-TSC Based SVC for Reactive Power Compensation and Harmonic Reduction in Unbalanced Distribution System. IET Generation, Transmission \& Distribution. 2018; 12(3): 571-585.

[4] N. A. M. Kamari, I. Musirin, Z. Othman, S. A. Halim. PSS Based Angle Stability Improvement Using Whale Optimization Approach. Indonesian Journal of Electrical Engineering and Computer Science. 2017; 8(2): 382-390.

[5] H. E. Keshta, A. A. Ali, E. M. Saied, F. M. Bendary. Application of Static Var Compensator (SVC) With PI Controller for Grid Integration of Wind Farm Using Harmony Search. International Journal of Emerging Electric Power Systems. 2016; 17(5): 555-566.

[6] N. A. Mohamed Kamari, I. Musirin, M. M. Othman. IPSO based SVC-PID for angle stability enhancement. International Journal of Simulation: Systems, Science and Technology. 2017; 17(41): 20.1-20.7

[7] A. F. A. Kadir, A. Mohamed, H. Shareef, M. Z. C. Wanik. Optimal Placement and Sizing of Distributed Generations in Distribution Systems for Minimizing Losses and THDv Using Evolutionary Programming. Turkish Journal of Electrical Engineering and Computer Sciences. 2013; 21: 2269-2282.

[8] A. A. Ibrahim, A. Mohamed, H. Shareef, S. P. Ghoshal. Optimal Power Quality Monitor Placement in Power Systems Based on Particle Swarm Optimization and Artificial Immune System. 3rd Conference on Data Mining and Optimization. Putrajaya. 2011: 141-145.

[9] Adadad B. Bentouati, L. Chaib, S. Chettih. A Hybrid Whale Algorithm and Pattern Search Technique for Optimal Power Flow Problem. 8th International Conference on Modelling, Identification and Control. Algiers. 2016: 10481053.

[10] P. Kundur. Power System Stability and Control. New York: McGraw-Hill, 1994: 766-775.

[11] M. H. M. Zaman, M. M. Mustafa, A. Hussain. Critical Equivalent Series Resistance Estimation for Voltage Regulator Stability Using Hybrid System Identification and Neural Network. International Journal on Advanced Science, Engineering and Information Technology. 2017; 7: 1381-1388.

[12] J. A. Ali, M. A. Hannan, A. Mohamed. Improved Indirect Field-Oriented Control of Induction Motor Drive Based PSO Algorithm. Jurnal Teknologi. 2016; 78(6-2): 27-32.

[13] L. A. Wong, H. Shareef, A. Mohamed and A. A. Ibrahim, An Enhanced Opposition-based Firefly Algorithm for Solving Complex Optimization Problems, Jurnal Kejuruteraan. 2014; 26: 89-96.

[14] N. N. Islam, M. A. Hannan, A. Mohamed, H. Shareef. Damping Power System Oscilaation Using Elitist Differential Search Algorithm in Multi Machine Power System. Journal of Theoretical and Applied Information Technology. 2016; 93(1): 41-48. 